\title{
Metodologia de Avaliação de Mundos Virtuais Educacionais: uma proposta segundo critérios tecnológicos e pedagógicos
}

\author{
Andressa Falcade - UFSM - andressafalcade@gmail.com \\ Aliane Krassmann - UFSM - alkrassmann@gmail.com \\ Fabrício Herpich - UFSM - fabricio.herpich@gmail.com \\ Laís Falcade - UFSM - laisfalcade@hotmail.com \\ Lisandra Manzoni Fontoura - UFSM - lisandramf@gmail.com \\ Roseclea Duarte Medina - UFSM - roseclea.medina@gmail.com
}

\begin{abstract}
Resumo: Este artigo apresenta um levantamento sobre ambientes virtuais tridimensionais educacionais que estão sendo desenvolvidos, bem como critérios avaliativos, apresentado uma proposta de metodologia para a avaliação de mundos virtuais, segundo critérios tecnológicos $e$ pedagógicos. A validação foi realizada no ambiente TCN5, expondo uma avaliação positiva da aplicação. O resultado do questionário pode ser considerado de confiança, segundo o coeficiente Alfa de Cronbach, que diagnosticou o mesmo como aceitável.
\end{abstract}

Palavras-chave: mundos virtuais educacionais, metodologia de avaliação, aprendizagem mediada por tecnologia, metaversos educacionais.

\section{Assessment Methodology of Educational Virtual Worlds: a proposal according to technological and pedagogical criteria}

\begin{abstract}
This paper presents a survey about three-dimensional educational virtual environments being developed, as well as its evaluation criteria, presenting a methodology for the evaluation of virtual worlds, according to technological and pedagogical criteria. The validation was performed in TCN5 environment, exposing a positive evaluation of the application. The result of the questionnaire can be considered reliable according to Cronbach's alpha coefficient, which has diagnosed it as acceptable.
\end{abstract}

Keywords: educational virtual worlds, evaluation methodology, technologymediated learning, educational metaverses.

\section{Introdução}

A tecnologia, hoje, nos permite viver uma vida à parte dentro do computador, seja ela para entretenimento, com os jogos em três dimensões (3D), seja para aprendizagem, através dos serious games - jogos sérios.

Utilizar os mundos virtuais (MV) para a construção da aprendizagem é aliar a facilidade tecnológica às estratégias e metodologias educacionais como uma das formas de facilitar a evolução da escola perante a sociedade informatizada. Porém, ainda existem poucas sugestões de avaliação de utilização de mundos virtuais.

Os mundos virtuais ou metaversos, utilizados na educação estão sendo construídos com enfoques particulares para o aprendizado de conteúdos específicos, porém a avaliação desses mundos ainda ocorre de forma empírica, através da observação da utilização dos MV pelos seus usuários (Bouda, 2012). Portanto, essa pesquisa tem como objetivo auxiliar na criação de uma proposta metodológica 
avaliativa de MV. Para tanto, estão sendo investigados processos de avaliação de ferramentas de uso pedagógico e critérios já levantados por outros autores sobre o tema. Este artigo se divide da seguinte forma: na Seção 2 serão expostos alguns conceitos sobre os mundos virtuais e algumas ferramentas que apoiam o seu desenvolvimento. $\mathrm{Na}$ Seção 3 serão apresentados alguns exemplos de metaversos e mundos virtuais educacionais. Na Seção 4 serão discutidos alguns critérios pedagógicos e tecnológicos de avaliação de aprendizagem e MV. Na Seção 5 será descrita a proposta de avaliação de MV formulada nesta pesquisa. Na Seção 6 serão apresentadas a validação e as discussões da pesquisa e, finalmente, na seção 7 o presente trabalho será finalizado com as considerações finais.

\section{Mundos virtuais e suas plataformas de desenvolvimento}

Um MV é uma simulação de um ambiente real, ou a formulação de um ambiente imaginário, fictício, criado para convivência e comunicação entre pessoas representadas por avatares que realizam ações e interagem entre si. (Backes, 2012).

Já Nelson e Erlanderson (2012) falam que MV são espaços com coordenadas em três dimensões onde o usuário movimentará o avatar que o representa, explorando o ambiente sozinho ou acompanhado de outros usuários.

Entretanto, Mueller et al. (2011) é mais específica em sua definição dos MV, pois diz que o mundo precisa facilitar a interação de muitos usuários, mesmo distantes geograficamente. Essa interação deve ser síncrona, para que os usuários interajam em tempo real; o MV deve ter várias formas de comunicação incluindo voz, textos e em linguagem corporal; o usuário deve ser representado por um avatar; os mundos devem ter duas propriedades que são fisicalidade e persistência, a primeira faz referência ao contexto de interação 3D compartilhada e a segunda ressalta que o ambiente deve continuar funcionando, mesmo que o usuário não esteja usando e, por fim, o programa deve lembrar a localização de pessoas e coisas bem como a posse de objetos.

O mundo virtual é a visualização em três dimensões de uma simulação do mundo real. Porém, para que ele seja criado, é necessária a utilização de uma plataforma de desenvolvimento (Opensimulator) ${ }^{1}$. Dentre as plataformas mais usadas estão o Second Life (SL) e o Open Simulator (OpenSim).

O SL é um mundo virtual criado em 2003, pela Linden Lab, sediada em San Francisco, EUA, e foi criado para o entretenimento e interação social (Second Life) ${ }^{2}$. Para uso educacional o SL possui diversos recursos como chat, com texto e voz, que podem ser gravados para posterior visualização assíncrona; tele transporte para outros locais do mundo; displays para demonstração de conteúdos e apresentação de slides; ferramentas de câmera para tirar fotos; uso de áudio e vídeo por streaming; permite a navegação em páginas web e a integração com o ambiente virtual de aprendizagem Moodle, através do mashup SLoodle. (Mattar Neto, 2008). Segundo a Association of Virtual Worlds, em 2008 o SL possuía 250 mundos virtuais e 16 milhões de contas criadas (Correia, Eiras, 2009).

O OpenSim é uma ferramenta open source escrita em linguagem C\#, criada em 2007, suporta ambientes online em 3D com tamanhos variáveis dentro da mesma instância, além de muitos usuários que podem acessar por diferentes protocolos e criar seus próprios conteúdos tridimensionais em tempo real. Ávila et al. (2013) ressalta como pontos positivos que o metaverso não exige muito conhecimento da pessoa que irá manuseá-lo, além disso, aceita diferentes formatos de arquivo, tanto os resultantes de ferramentas de modelagem 3D com extensão .dae, quanto arquivos com extensão .xml resultantes de repositórios online. O Opensim possui alta compatibilidade com o SL, 
porém é menos restrito por ser totalmente gratuito, não limitando o número de objetos e o tamanho de scripts (Wagner et al., 2013).

\section{Exemplos de Alguns Mundos Virtuais Educacionais}

A seguir serão apresentados alguns projetos que buscam utilizar mundos virtuais na educação, destacando a ocorrência de processos avaliativos durante a aplicação dos mesmos, bem como a aprendizagem mediada por ele.

O mundo TCN5, utilizado neste estudo como ferramenta base da avaliação, conforme Voss (2014) é um ambiente virtual imersivo desenvolvido para o ensino de Redes de Computadores (RC) que trata informações de contexto do aluno (e.g., tecnologia, estilo cognitivo e qualidade do contexto), proporcionando ao mesmo uma aprendizagem significativa, a partir da integração e interação entre usuários e tecnologia.

Sua construção teve como base quatro elementos principais: WampServer, OpenSim, Sloodle e o Moodle UVLEQoC (Ubiquitous Virtual Learning Environment with Quality of Context), que agrega as características tanto dos módulos U-SEA 2.0 (Sistema de Ensino Adaptado Ubíquo) e SEDECA 2.0 A (Sistema para Diagnosticar Estilos de Aprendizagem) como do Tema Bootstrap, aplicando parâmetros e métricas de QoC (Quality Of Context) sobre as informações coletadas no ambiente, para garantir que o contexto formulado esteja adequado às preferências e necessidades dos alunos.

Foi validado em um estudo Não-Experimental utilizando uma Arquitetura Pedagógica, em uma turma de 25 alunos da disciplina de RC do Curso de Ciência da Computação da UFSM. Os resultados da avaliação com os alunos atenderam as expectativas, alcançando uma classificação "Excelente" na escala do teste de usabilidade SUS (System Usability Scale), bem como um aumento considerável na média obtida na elaboração dos mapas conceituais após a utilização do TCN5, um indício de que o ambiente auxiliou na construção do conhecimento dessa turma.

Já Silva e Felix (2011), utilizaram um MV, criado no OpenSim, como auxílio à disciplina de Oficina de Projeto Teórico do curso técnico em Design de móveis do IF Sul-Rio-Grandense. O objetivo era avaliar a interação dos alunos com o ambiente e com as atividades propostas, que envolviam a projeção de móveis em três dimensões. As aulas ocorreram somente através do MV, inclusive as interações e questionamentos sobre as funcionalidades do mesmo e sobre a disciplina em si.

O que se verificou foi o interesse dos estudantes e mesmo da professora na utilização da ferramenta como prática das aulas de projeto. Como dificuldade, Silva e Felix (2011) comentam o fato de o ambiente ser igual para todos, sem separação, podendo levar à fuga do objetivo da aula.

Houve uma análise sobre a interação dos alunos com o ambiente. No geral, a conclusão foi positiva, pois através da interação dentro do mundo, todos tinham acesso às dúvidas e às soluções apesentadas para elas, comentadas tanto pelas professoras quanto pelos próprios alunos, gerando a aprendizagem colaborativa. Outro ponto importante ressaltado pelos autores foi que o papel do professor mudou, não sendo mais o detentor do conhecimento, mas o incentivador da construção do conhecimento do estudante.

Outro MV existente é o Campus 3D do IF de Alagoas (IFAL) desenvolvido com ferramentas que podem ser utilizadas com objetivo educacional, como fóruns, links, chats e projeção de slides. Ele foi utilizado por dez alunos do curso de Sistemas de Informação do IFAL, abordando funcionalidades e características, como se movimentar, conversar com colegas através do chat, visualizar conteúdos, realizar atividades. 
A avaliação do curso, pelos alunos, identificou grande dificuldade de acesso fora do laboratório da universidade, pois a utilização do ambiente exige conexão com Internet banda larga e hardware suficiente para execução. Apesar disso, Freire et al. (2010) acreditam que a interação entre alunos e professores ocasionada dentro do ambiente facilita a aprendizagem, por isso pretendem implantar a sua utilização para o estudo de outros conteúdos e por outros cursos, não somente o de informática. Pretendem, também, através dessa implantação, testar todas essas ferramentas disponibilizadas para a aprendizagem, como verificação da sua efetividade no objetivo de auxiliar a construção do conhecimento.

O laboratório virtual VEJA (Virtual Environment for Geometry Acquaintance) (Tarouco et al., 2012), também exemplo neste estudo, foi construído pela UFRGS com o OpenSim e tem, como objetivo, o estudo da geometria e é composto por cinco salas, uma de nivelamento, outra de experimentação inicial com os objetos geométricos, outra de construção de objetos, onde o aluno poderá aplicar os seus conhecimentos, outra sala que é um laboratório de ensaio para aplicação prática de suas experiências e uma área de convivência para a interação com os outros usuários do ambiente. As salas são ligadas por uma área de circulação que permite ao aluno avançar ou retroceder de acordo com a sua necessidade, não existindo uma sequência a ser seguida. A criação do laboratório VEGA teve por culminância uma proposta de roteiro pedagógico para a utilização do mesmo.

Os autores refletem que a utilização de mundos virtuais na educação auxilia na construção do conhecimento por disponibilizar a interação social, a "aprendizagem ativa" como sendo a modificação do ambiente e dos personagens e a resolução de problemas caracterizada pela simulação de conhecimentos reais no mundo virtual.

\section{Mundos Virtuais e Avaliação}

Utilizar MV como uma nova ferramenta de auxílio à construção do conhecimento pode ser muito positivo, porém eles, por si só, não promovem a aprendizagem (Crisp, 2012). Nelson e Erlanderson (2012) falam que na concepção de mundos virtuais a avaliação assume duas formas, a primeira é com relação à qualidade do software associado à experiência do usuário e a segunda é relacionado à forma de aprendizagem do aluno referente a utilização do mundo virtual.

A seguir serão apresentados alguns critérios pedagógicos e tecnológicos de avaliação da qualidade de mundos virtuais para a aplicação na educação, abordados por Crisp (2012), Barbosa et. al. (2005), Barbosa et al. (2005), Bouda (2012), Revees e Harmon (1996) citados por Godói e Padovani (2008) e Nelson e Erlanderson (2012), como forma de embasamento para a formulação de uma proposta metodológica avaliativa mista de MV.

\subsection{Critérios pedagógicos de avaliação da aprendizagem}

Os mundos virtuais são capazes de criar ambientes de aprendizado e de avaliação dentro da escola, desde que sejam formulados com objetivos pedagógicos e as atividades de aprendizagem e tarefas de avaliação associados estejam alinhados com as habilidades e capacidades que devem ser desenvolvidas no âmbito do curso (Crisp, 2012).

Como Nelson e Erlanderson (2012) comentam, a avaliação do mundo virtual está amplamente relacionada com a avaliação da aprendizagem que foi mediada por ele. Para avaliar a aprendizagem dentro de MV podem ser utilizadas ferramentas de apoio pedagógico encontrado dentro do próprio ambiente tridimensional, como wikis, blogs, fóruns de discussão e portfólio. Existem ferramentas que proporcionam a realização de testes como o Quiz HUD ${ }^{3}$ e o Sloodle (Crisp, 2012). 
O Sloodle é uma ferramenta gratuita que permite integrar mundos virtuais do SL e do OpenSim ao Moodle (Sloodle) ${ }^{4}$, o que facilita a utilização dos diversos recursos contidos no AVA voltados à avaliação da aprendizagem, como chats, wikis, fóruns de discussão, questionários, blogs e e-portfólios (Crisp, 2012).

Outra forma de avaliar o processo de aprendizagem é através da técnica desenvolvida por Josep Novak, que trata da organização do conhecimento pelo mapeamento dos saberes demonstrados por mapas conceituais (Barbosa et al., 2005). Um dos objetivos da avaliação por mapas conceituais é conhecer a mudança da estrutura cognitiva do estudante com relação ao conteúdo estudado (Araújo et al., 2002).

\subsection{Critérios tecnológicos de avaliação do mundo virtual}

Segundo Bouda (2012) o desenvolvimento do mundo virtual deve ter o design centrado no usuário. Além disso, deve ser construído de tal forma a ressaltar primeiro as informações mais importantes, atentando para informações que desviem a atenção do usuário do objetivo a ser realizado. $\mathrm{O}$ autor apresenta uma metodologia de avaliação de mundos empírica, dada através da observação da interação do estudante com o ambiente imersivo, onde se cria um roteiro de utilização para resolução de algumas tarefas.

Nesta metodologia de avaliação observa-se a reação do usuário quanto à interface que deve ter três características fundamentais (Bouda, 2012): eficácia, que verifica se as metas previstas de utilização são alcançadas; eficiência, que diz respeito à quantidade de recursos que devem ser utilizados para alcançar os objetivos; e satisfação, que mede o quanto o sistema global é aceitável. Para realizar essa observação, é determinado um roteiro a ser seguido e é medido o tempo necessário para a conclusão da tarefa, bem como a quantidade de erros cometidos durante esse tempo. Após essa medida quantitativa, o ambiente é exposto a uma revisão para melhorar alguns aspectos identificados na observação qualitativa e submetido a novo teste para comparação com os resultados quantitativos obtidos na primeira fase (Bouda, 2012).

Em consonância com Bouda (2012), Revees e Harmon (1996) citados por Godói e Padovani (2008), a importância de avaliar critérios como a facilidade de utilização e de navegação e aspectos da carga cognitiva se referem a três critérios: 1. A quantidade de informação que a interface apresenta ao usuário. 2. O design de tela, que faz referência às informações relevantes nos lugares certos. 3. A apresentação da informação, que deve ser organizada e clara ao estudante. Ressaltam também a estética do ambiente podendo ser avaliada como agradável ou não agradável, e a integração entre as mídias, que avalia se o programa permite a utilização de diversas mídias e se a funcionalidade geral do programa apresenta um padrão com relação à interface e aos critérios pedagógicos.

Uma característica importante comentada por Revees e Harmon (1996), citados por Godói e Padovani (2008), é a capacidade de o mundo virtual mapear os caminhos percorridos pelo usuário como forma de identificar problemas de utilização e aprendizado dos cenários apresentados pelo ambiente.

\section{Proposta de Avaliação de Mundos Virtuais}

A proposta metodológica desta pesquisa teve como principal objetivo formular um questionário unindo os critérios tecnológicos aos pedagógicos, de forma avaliar o $\mathrm{MV}$ como um todo, de maneira mais completa e eficaz. Para tanto, foram tomadas como base as produções de Crisp (2012), Bouda (2012), Revees e Harmon (1996) apud Godói e Padovani (2008) e Mozzaquatro (2010), adaptando as questões levantadas anteriormente a fim de identificar algumas das características que podem interferir na utilização desses ambientes pelos seus usuários. 
Entre as questões está o tempo de espera para o carregamento de todas as funcionalidades do $\mathrm{MV}$, levando em consideração a velocidade de rede e o hardware que o usuário possui, o que pode ser um fator negativo na utilização do ambiente para o aprendizado. Outro fator a se destacar é o tempo que o usuário leva de um local a outro dentro do metaverso, e se há uma ferramenta de teletransporte para agilizar o deslocamento quando esses locais se encontram muito distantes.

Outra questão apontada é a possibilidade de integração do MV com o Moodle, proposta pelo Sloodle, que proporciona maior interação e consequentemente maior interesse no aprendizado por parte dos alunos, consequência da aproximação da educação ao seu linguajar. (Pereira e Rocha, 2014). Importante também foi a questão apresentada sobre qual o formato reconhecido pelo chat: texto, voz ou ambos, e se essas conversas podem ser salvas para serem vistas e ouvidas depois. Essa característica é fundamental, pois se o estudante possuir material explicativo à sua disposição para estudo, seu aprendizado pode ser fortalecido. Funcionalidades como visualizar o material didático do Moodle de dentro do MV, abrir imagens postadas e ouvir os sons disponíveis no metaverso é básico na tentativa de fomentar o interesse do estudante pela ferramenta.

Adaptar a forma de apresentar as informações e conteúdos aos estilos cognitivos fazem os alunos aprender mais e melhor segundo Mozzaquatro (2010), a qual relata que os indivíduos possuem diferentes formas de processar e organizar conhecimentos e isso interfere no aprendizado, por isso a importância de questionar se há ou não essa adaptação.

De forma geral, o questionário sugerido neste trabalho é composto por vinte e quatro questões, sendo vinte e duas delas perguntas fechadas (com três alternativas de escolha) e duas questões abertas (com possibilidade de dissertar sobre o que foi perguntado).

As perguntas fechadas se dividem em três categorias: 1. Uma questão diagnóstica para realizar o levantamento da familiaridade dos testadores com o ambiente, critério que influencia nas respostas segundo Nelson e Erlanderson (2012). 2. Vinte questões que se detém na avaliação do metaverso TCN5, disponibilizado para validar o instrumento avaliativo proposto. 3. Refere-se à opinião dos participantes quanto à metodologia de avaliação por eles analisada.

Já as perguntas abertas tiveram por objetivo levantar as análises críticas dos participantes quanto à aplicação do MV TCN5 em um ambiente de aprendizagem, além de possíveis melhorias no instrumento de avaliação. O questionário, na íntegra, está disponível no serviço de armazenamento e sincronização de dados Google Drive ${ }^{5}$.

Para quantificar e avaliar os resultados tomou-se como base a escala Likert de avaliação (Cunha, 2007), que traz níveis de manifestação do usuário e o coeficiente Alfa de Cronbach (Herpich et al., 2014). A escala Likert propõe declarações que recebem um número específico de respostas e estas recebem um valor pré-determinado para que se possa quantificar a avaliação do objeto proposto através do questionário (Nunes et. al., 2013). Neste caso, o questionário voltou-se a questões sobre a opinião do testador quanto à metodologia de avaliação. Se utilizaram as medidas "não", "parcialmente" e "sim", que receberam, respectivamente, os valores 0 (zero), 2,5 (dois e meio) e 5 (cinco). Na questão diagnóstica foram utilizadas as alternativas "baixa", "média" e "alta" com os mesmos valores, respectivamente.

\section{Validação e discussão dos resultados da pesquisa}

A validação do questionário foi realizada por dois alunos do curso de graduação em Ciência da Computação e cinco alunos do curso de Pós-Graduação, em nível de 
mestrado, em Ciência da Computação da UFSM. Para tanto, foi disponibilizado um usuário e senha padrão para acesso ao MV.

A questão diagnóstica mostrou que três dos sete usuários têm alta familiaridade com o mundo virtual TCN5. O restante se dividiu igualmente entre baixa e média familiaridade.

Os resultados apontam para identificação de importantes características acerca da utilização do TCN5. Entre elas estão:

- Seis dos usuários consideraram que o MV não é lento de utilizar e notaram a existência de um instrumento de teletransporte;

- Quatro dos usuários percebeu a existência do mapeamento de passos;

- Quatro deles disseram que o chat permite a conversação utilizando a fala, porém não permite salvar essas conversas para análise posterior;

- Cinco participantes responderam positivamente quanto à facilidade de visualização de conteúdos e materiais de aula dentro do mundo virtual e quatro responderam o mesmo para a visualização dos conteúdos dispostos dentro do AVA Moodle através do MV;

- Cinco dos testadores disseram ser possível acessar uma página web externa através do ambiente tridimensional.

- Três pessoas disseram receber feedback parcial das atividades concluídas e das que ainda estão por finalizar. Já outros três disseram que o MV não sinaliza as tarefas que estão por realizar.

- Seis dos usuários relataram que o sistema de som do MV facilita total ou parcialmente o sentimento de imersão no ambiente 3D

- Cinco participantes disseram que a interface do mesmo chama a atenção para a sua utilização, apesar de considerarem o cenário parcialmente completo.

As questões de interação mostraram que mais de cinco dos usuários acharam fácil interagir com outros usuários e o ambiente tridimensional, além de criar e modificar objetos dentro dele e a maioria achou fácil ou parcialmente fácil modificar as características de seus avatares.

Sobre a existência da separação dos conteúdos de acordo com estilos cognitivos de aprendizagem, teve-se um empate nas alternativas "sim" e "parcialmente", onde cada uma obteve três sinalizações enquanto a alternativa "não" obteve apenas uma sinalização. $\mathrm{O}$ indicio aqui, pode representar que o usuário não reconhece/diferencia seu estilo ou o TCN5 está tão adaptado ao estilo do usuário que o mesmo não sente a diferenciação do conteúdo.

A última questão, ainda sobre o mundo tridimensional pergunta a opinião dos usuários sobre a aplicabilidade do TCN5 em um ambiente educacional real, sendo que seis dos os usuários consideraram que o mesmo facilitaria a aprendizagem dos estudantes. Segundo a opinião dos usuários esta constatação é possível devida proximidade com o mundo real, sem os riscos e custos inerentes dessa aplicação.

Ao final foi colocada uma questão sobre a opinião dos participantes quanto à eficiência do questionário na avaliação do MV, sendo que eles se dividiram igualmente entre as alternativas "sim" e "parcialmente".

A interrogativa sobre a validade do questionário vai ao encontro com o coeficiente $\alpha$ de Cronbach calculado a partir dos resultados obtidos pela Escala Likert, demonstrando que o questionário alcançou a nota 0,756 correspondendo ao nível de confiança "aceitável" segundo George e Mallery (2003) citado por Herpich et al.(2014). A Figura 1 apresenta os cálculos e os dados utilizados para determinar o valor do coeficiente $\alpha$. 


\begin{tabular}{|c|c|c|c|c|c|c|c|c|c|c|c|c|c|c|c|c|c|c|c|c|c|c|c}
$\begin{array}{c}\text { Questão/ } \\
\text { Usuário }\end{array}$ & Q1 & Q2 & Q3 & Q4 & Q5 & Q6 & Q7 & Q8 & Q9 & Q10 Q11 Q12 Q13 Q14 Q15 Q16 Q17 Q18 Q19 Q20 Q21 Q22 & $\begin{array}{c}\text { Soma das } \\
\text { respostas }\end{array}$ \\
\hline Usuário 1 & 5 & 0 & 5 & 5 & 5 & 5 & 5 & 5 & 2,5 & 0 & 2,5 & 5 & 5 & 2,5 & 2,5 & 5 & 5 & 5 & 2,5 & 5 & 5 & 5 & 87,5 \\
\hline Usuário 2 & 0 & 0 & 0 & 2,5 & 2,5 & 5 & 5 & 5 & 2,5 & 2,5 & 2,5 & 5 & 2,5 & 5 & 2,5 & 5 & 5 & 5 & 2,5 & 5 & 5 & 2,5 & 72,5 \\
\hline Usuário 3 & 5 & 0 & 5 & 2,5 & 5 & 2,5 & 5 & 5 & 5 & 0 & 5 & 5 & 5 & 5 & 0 & 5 & 2,5 & 2,5 & 2,5 & 5 & 5 & 5 & 82,5 \\
\hline Usuário 4 & 2,5 & 2,5 & 5 & 2,5 & 5 & 5 & 5 & 5 & 5 & 0 & 0 & 5 & 5 & 0 & 0 & 5 & 2,5 & 5 & 2,5 & 2,5 & 2,5 & 2,5 & 70 \\
\hline Usuário 5 & 2,5 & 0 & 5 & 5 & 5 & 5 & 5 & 5 & 5 & 0 & 5 & 5 & 5 & 5 & 5 & 0 & 2,5 & 5 & 2,5 & 5 & 5 & 5 & 87,5 \\
\hline Usuário 6 & 5 & 0 & 5 & 5 & 5 & 5 & 5 & 5 & 5 & 0 & 5 & 5 & 5 & 2,5 & 0 & 5 & 5 & 0 & 0 & 5 & 5 & 2,5 & 80 \\
\hline Usuário 7 & 0 & 0 & 5 & 5 & 2,5 & 0 & 5 & 2,5 & 2,5 & 2,5 & 2,5 & 2,5 & 2,5 & 2,5 & 2,5 & 0 & 0 & 0 & 0 & 2,5 & 5 & 2,5 & 47,5 \\
\hline variância & 4,3 & 0,8 & 3,1 & 1,5 & 1,3 & 3,3 & 0 & 0,8 & 1,5 & 1,3 & 3,1 & 0,8 & 1,3 & 3,1 & 3,1 & 5,1 & 3,1 & 4,8 & 1,3 & 1,3 & 0,8 & 1,5 & 168,6 \\
\hline
\end{tabular}

Figura 1 - Resultados do cálculo do coeficiente $\alpha$.

Ainda no questionário, foi disponibilizado um local para que os participantes dessem sugestões de melhoria para a metodologia de avaliação. Essas sugestões estão descritas a seguir:

- Formulação de um questionário mais geral sobre as funcionalidades dos mundos virtuais;

- Implantação de uma questão que demonstrasse o nível de conhecimento dos avaliadores sobre o assunto;

- Inclusão de uma questão que interrogasse a existência da funcionalidade drag-and-drop (arrastar e largar) de objetos para dentro do MV;

- Existência de uma questão aberta que proporcionasse aos testadores sugerir roteiros de utilização;

- Inserção de uma alternativa "Não sei" ou "não se aplica" para o caso de não saber responder a uma questão de forma exata, por não conhecer o ambiente de teste.

\section{Considerações Finais}

Com este trabalho pode-se refletir sobre uma possível forma de avaliação dos mundos virtuais, aplicando um procedimento avaliativo no MV TCN5. Buscou-se, com isso, salientar a importância de se criar uma metodologia mais formal e concreta, que faça o diagnóstico de melhorias a serem implementadas nos ambientes virtuais imersivos.

A educação busca se atualizar a cada dia mais, através de suas ferramentas, para melhorar a aprendizagem. Porém, carecem mecanismos de avaliação para nortear a contribuição tecnológica na formação do saber e melhoria no processo ensinoaprendizagem. Dentro desse contexto, essa pesquisa trouxe uma proposta metodológica de avaliação que pode ser utilizada no desenvolvimento de metaversos educacionais, identificando critérios norteadores que contribuam na tarefa de auxiliadores da construção e busca do conhecimento.

A metodologia, baseada na escala Likert, foi construída para identificar características tecnológicas, como recursos e ferramentas, a integração com o AVA Moodle através do Sloodle, características operacionais como facilidade de abrir e visualizar diferentes tipos de materiais e o feedback de atividades e características pedagógicas como a adaptação de estilos cognitivos de aprendizagem. O questionário foi quantificado segundo o coeficiente $\alpha$ de Crombach, diagnosticando o mesmo como sendo "aceitável", concluindo, assim, que os resultados aqui apresentados são relevantes e confiáveis.

Como projeto futuro, pretende-se melhorar a metodologia de avaliação de mundos virtuais, ampliando os critérios apresentados, incluindo as sugestões apontadas através desta pesquisa e considerando outras metodologias de avaliação existentes. Essa 
proposta serve com sugestão de instrumento de avaliação de MV educacionais a outros autores, que busquem verificar a eficácia de seus metaversos.

${ }^{1}$ OPENSIMULATOR. Disponível em $<$ http://goo.gl/721E2l $>$

${ }^{2}$ SECONDLIFE, Disponível em $<$ http://goo.gl/yplR1D $>$

${ }^{3}$ QUIZHUDE. Disponível em $<$ http://goo.gl/M1e2ht $>$

${ }^{4}$ SLOODLE. Disponível em $<$ http://goo.gl/LF7i1L $>$

${ }^{5}$ QUESTIONÁRIO. Disponível em $<$ http://goo.gl/Rptd9B $>$

\section{Referências}

ARAÚJO, A. M. T; MENEZES, C. S.; CURY, D.. Um Ambiente Integrado para Apoiar a Avaliação da Aprendizagem Baseado em Mapas Conceituais. In XIII Simpósio Brasileiro de Informática na Educação. 2002. Disponível em $<$ http://goo.gl/ZZH1Ni> Acesso em 07 de maio de 2014.

ÁVILA, B.; AMARAL, É.; Tarouco, L.. Implementação de Laboratórios Virtuais no metaverso OpenSim. Revista Renote, Novas Tecnologias na Educação. Vol. 11, n. 1. XXI Ciclo de Palestras sobre Novas Tecnologias na Educação. Porto Alegre, RS, 2013. Disponível em < http://goo.gl/AOik1D> Acesso em 15 de abril de 2014.

BACKES, L.. As manifestações da autoria na formação do educador em espaços digitais virtuais. Revista de Educação, Ciência e Cultura. vol. 17, nº. 2, jul./dez. 2012. Disponível em $<$ http://goo.gl/jGDcYY $>$ Acesso em 10 de maio de 2014.

BARBOSA, M. L.; ALVES, Á. S.; JeSUS, J. C. O.; BURNHAM, T. F.. Mapas conceituais na avaliação da aprendizagem significativa. In XVI Simpósio Nacional do Ensino da Física. 2005. Disponível em <http://goo.gl/TLiaBL> Acesso em 07 de maio de 2014.

BOUDA, T. User-centered Design and Evaluation of Virtual Worlds. 2012 . In Experiential Learning in Virtual Worlds: 2nd Global Conference, Prague (Czech Republic). Disponível em < http://goo.gl/MyyNiM > Acesso em 10 de maio de 2014.

CORREIA, M. M.; EIRAS, B. D.. Mundos Virtuais: que vida existe no Second Life? Cadernos BAD, Revista da associação portuguesa de bibliotecários, arquivistas e documentalistas, $\mathrm{n}^{\mathrm{o}}$ 1/2, 2009-2010. Páginas 70-83. Disponível em $<$ http://goo.gl/T3jtc4> Acesso em 22 de abril de 2014.

CRISP, G.. Meaningful assessment within virtual worlds - what should it look like? 2012 . In Experiential Learning in Virtual Worlds: 2nd Global Conference, Prague (Czech Republic). Disponível em <http://goo.gl/TBIu94>Acesso em 10 de maio de 2014.

CUNHA, L. M. A.. Modelos Rasch e Escalas de Likert e Thurstone na medição de atitudes. Dissertação. Mestrado em Probabilidades e Estatística. Universidade de Lisboa, Faculdade De Ciências, Departamento de Estatística e Investigação Operacional. 2007. Disponível em <http://goo.gl/NgcO1l> Acesso em 10 de maio de 2014.

FREIRE, A.; ROLIM, C.; BESSA, W.. Criação de um Ambiente Virtual de EnsinoAprendizagem usando a plataforma opensimulator. V Congresso de Pesquisa e Inovação da Rede Norte Nordeste de Educação Tecnológica (V CONNEPI). MaceióAL. 2010. Disponível em <http://goo.gl/gWCAsB> Acesso em 22 de abril de 2014.

GODOI, K. A.; PADOVANI, S.. Avaliação de objetos de aprendizagem: um estudo sobre abordagens e critérios de avaliação. $3^{\circ}$ CONAHPA, Congresso Nacional de Ambientes Hipermídia para Aprendizagem. São Paulo: SP, 2008. Disponível em $<$ http://goo.gl/ZkzGOj> Acesso em 16 de abril de 2014. 
HERPICH, F.; JARDIM, R. R.; NUNES, F. B.; VOSS, G. B.; FONTOURA, L. M.; MEDINA, R. D.. Laboratorio Virtual: uma Ferramenta Imersiva para Auxilio no Ensino de Engenharia de Software. XVI Symposium on Virtual and Augmented Reality, 2014.

MATTAR NETO, J. A.. O uso do Second Life como ambiente virtual de aprendizagem. GT2, Jogos eletrônicos e Educação. Seminário. 2008. Disponível em $<$ http://goo.gl/J8GBXm > Acesso em 08 de maio de 2014.

MOZZAQUATRO, P. M.. Adaptação do Mobile Learnig Engine Moodle (MLE Moodle) aos diferentes estilos cognitivos utilizando hipermídia adaptativa. Dissertação de Mestrado. Programa de Pós-Graduação em Informática, Universidade Federal de Santa Maria. 2010. Disponível em <http://goo.gl/if6NaT> Acesso em 12 de maio de 2014.

MUELLER, J.; HUTTER, K.; FUELLER ,J.; MATZLE, K.. Virtual worlds as knowledge management platform - a practice-perspective. Info Systems $\mathrm{J} \mathrm{n}^{\mathbf{0}} 21$, 2011. Disponível em <http://goo.gl/dJnDZd $>$ Acesso em 09 de maio de 2014.

NELSON, B. C.; HERLANDSON, B. E. Design for Learning in Virtual Worlds. Library of Congress Cataloging-in-Publication Date. By Swales \& Willis Ltd. 2012. 229 páginas.

NUNES, F. B.; VOSS, G. B.; HERPICH, F.; MÜHLBEIER, A. R. K.; POSSOBOM, C. C.; MEDINA, R. D.. Viewers para ambientes virtuais imersivos: uma análise comparativa teórico-prática. Revista Renote, Novas tecnologias na Educação. V. 11 $\mathrm{N}^{\mathrm{o}}$ 1, julho, 2013.

PEREIRA, R. G.; ROCHA, R.. A nova fronteira da educação a distância, os mundos virtuais triimensionais: Moodle + Second Life $=$ Sloodle. Sistemas de Informação, FAMINAS-BH, Técnico em Tecnologia da Informação - UFMG. 2014. Disponível em $<$ http://goo.gl/FJpdIa $>$ Acesso em 12 de maio de 2014.

SILVA, A. C. R.; FÉLIX, N. R.. Mundos Virtuais Baseados em Plataformas Livres como Apoio ao Ensino do Processo Projetual. $5^{\circ}$ CONAHPA, Congresso Nacional de Ambientes Hipermídia para Aprendizagem. Pelotas: RS, 2011. Disponível em $<$ http://goo.gl/O8GCaC> Acesso em 20 de abril de 2014.

TAROUCO, L.; ÁVILA, B.; AMARAL, É.; ZEDNIK, H.. VEGA - Implementando um Laboratório Virtual Imersivo no OpenSim. Revista Renote, Novas Tecnologias na Educação. ISSN 1679-1916. Vol. 10, n. 1 (2012). XIX Ciclo de Palestras sobre Novas Tecnologias na Educação. Porto Alegre, RS, 2012. Disponível em $<$ http://goo.gl/gq2KK2> Acesso em 19 de abril de 2014.

VOSS, G. B.. TCN5 - Teaching computer networks in a free immersive virtual environment. Dissertação de mestrado. Mestrado em Ciência da Computação. Universidade Federal de Santa Maria, UFSM. 2014.

WAGNER, R.; PIOVESAN, S. D.; PASSERINO, L. M.; LIMA J. V. Desenvolvendo ambientes de realidade virtual utilizando OpenSim. LACLO 2013, Octava Conferencia Latinoamericana de Objetos y Tecnologías de Aprendizaje. Vol 4, $\mathrm{n}^{\mathrm{o}} 1$. Disponível em < http://goo.gl/JGJKAW> Acesso em 24 de abril de 2014. 